\section{(C) OPEN ACCESS}

\title{
Defining patient centricity with patients for patients and caregivers: a collaborative endeavour
}

\author{
Guy Yeoman, ${ }^{1}$ Patricia Furlong, ${ }^{2}$ Michael Seres, ${ }^{3}$ Helena Binder, ${ }^{4}$ \\ Helena Chung, ${ }^{5}$ Vincenzo Garzya, ${ }^{1}$ Rachel RM Jones ${ }^{6}$
}

\begin{abstract}
'AstraZeneca Pharmaceuticals, Melbourn, UK

2Patient Project Muscular Dystrophy, Hackensack, New Jersey, USA

${ }^{3} 11$ Health, Borehamwood, Hertfordshire, UK

${ }^{4}$ Corvista, Cheshire, UK ${ }^{5}$ AstraZeneca Pharmaceuticals, Gaithersburg, Maryland, USA ${ }^{6}$ Formerly AstraZeneca Pharmaceuticals, Macclesfield, UK
\end{abstract}

\section{Correspondence to} Guy Yeoman, AstraZeneca Pharmaceuticals, Da Vinci Building, Melbourn Science Park, Melbourn, Royston, SJ8 6HB UK Guy.Yeoman@astrazeneca.com

Received 14 September 2016 Revised 28 January 2017 Accepted 6 March 2017 Published Online First 24 March 2017

\section{CrossMark}

To cite: Yeoman $\mathrm{G}$, Furlong $\mathrm{P}$, Seres $\mathrm{M}$, et al. BMJ Innov 2017;3:76-83.

\begin{abstract}
Background Patient engagement is an essential aspect in the research/development of biopharmaceutical products and disease management. Improving the lives of patients requires a deep understanding of their medical conditions, experiences, needs and priorities. However, a consistent definition of patient centricity is lacking. A series of initiatives was conducted to define patient centricity and its important principles impacting the biopharmaceutical industry.
\end{abstract}

Methods Interviews, questionnaires and literature reviews were conducted involving key stakeholders to initially identify issues of importance to patients, healthcare providers and payers. Subsequently, two identical workshops which included 22 patients/carers created a definition of patient centricity and the healthcare values important to patients/caregivers. Outputs were tested in a validation exercise involving patients in predominantly US ( $n=470)$ and European $(n=703)$ patient forums.

Results Initial research provided deeper understanding of patient needs and key topics of interest that were used to cocreate a definition of patient centricity and 10 associated principles of importance to the biopharmaceutical industry.

Wider testing of these outputs among predominantly US/European patient communities confirmed their validity. Patient centricity should be defined as 'Putting the patient first in an open and sustained engagement of the patient to respectfully and compassionately achieve the best experience and outcome for that person and their family'. Important principles for patients focused on education/information, cocreation, access and transparency.

Conclusions The development of a consistent definition of patient centricity and its associated principles provides an opportunity for biopharmaceutical companies to adopt and use these as a reference point for consistent patient engagement throughout the product life cycle.

\section{INTRODUCTION}

The development of new medicines is undertaken to improve the health and outcomes of patients. However, until recently, patient involvement in biopharmaceutical development has often been infrequent, episodic and restricted to the periphery apart from direct participation in clinical trials and postapproval activities such as disease education. But why is this? Biopharmaceutical innovation and development is complex and highly regulated with well-defined but proscriptive technical requirements for the conduct of clinical research. ${ }^{12}$ As would be expected, much focus has been placed on ensuring patient safety, ${ }^{2}$ appropriate measurement of clinical outcomes, ${ }^{2}$ and more recently in establishing patient confidentiality. ${ }^{3}$ All of these aspects created a regulatory-focused process, ${ }^{2}$ orientated around healthcare providers, ${ }^{4}$ efficacy and safety of products and submission of data. ${ }^{2}$ Patient participation was always recognised but in a paternalistic way, with patients being instructed and not overburdened with information. ${ }^{4}$ It is easy to understand therefore that a culture has historically existed where research has been conducted 'on', 'about' or 'for' patients. ${ }^{5}$ Indeed, a clear disconnect has existed between the biopharmaceutical industry and the ultimate end user, the 'Patient', 6 with patient engagement often limited to clinical trial participation or education about a new drug and frequently using healthcare professionals as a proxy. $^{7}$ Therein lies a problem. Health outcomes are dependent on patient 
engagement, ${ }^{8}$ but the full benefits of pharmacotherapy cannot be realised when, for example, $\sim 50 \%$ of patients do not take their medication as prescribed in the long term. ${ }^{9}$ The causes of non-compliance are myriad and include patient factors (eg, forgetfulness, cost, inability to obtain medication), physician factors (eg, poor doctor-patient relationship, communication issues) and medication factors (eg, adverse events, complicated regimens). ${ }^{10}$ Furthermore, drug products may be developed that patients do not want and will not use. $^{7}$ Clearly, development of medicines using a patient-centric approach provides an opportunity to more closely meet patient needs and therefore improve their lives in ways that patients and their families view as meaningful. In this respect, improving the lives of patients and their loved ones requires a deep understanding of their medical conditions, real-world experiences, needs and priorities. ${ }^{8}$ Therefore, it is now essential that the biopharmaceutical industry partner with patients in the discovery, research, development, distribution and access to medicines to bring about outcomes that are meaningful to them. Working with patients fosters innovation and it will ensure that the objectives of patients are met early on in the biopharmaceutical development process by incorporating their views and needs, leading to more impactful patient outcomes. ${ }^{8}$ Patients are the ultimate end users of medicines and it is they and their carers that should prioritise their needs and identify the outcomes that they desire. In this respect, patients should be given the opportunity to define their needs, ${ }^{8}$ including the value of interventions, the benefit and risk trade-offs based on their values, their desired clinical outcomes, preferences and experiences.

This concept of 'patient centricity" ${ }^{\prime 1}$ and the opportunity to include patient-centred activities in drug development has been a key topic of interest in the past few years, ${ }^{6}$ 12-18 with parallel advances made with key stakeholders including industry, regulators and healthcare professionals. ${ }^{19-21}$ Patients are also demanding that drug development becomes more patient centric and that more patient-centric relationships are developed. ${ }^{22}{ }^{23}$ Indeed, this is a much discussed and written about topic, but as yet, a consistent definition of patient centricity as applied to drug development has not been published, and although definitions relating to other key stakeholders (eg, healthcare providers ${ }^{24}$ ) have been developed, these are more specific to the delivery of healthcare by healthcare professionals and not necessarily transferable to the drug development process. Indeed, patients to date have not been asked to define how they want to engage in the scientific research and development for their medicines of the future. Research has also shown that patient centricity is a highly recognised term among life science executives in the biopharmaceutical industry, but a consistent definition is lacking. ${ }^{25}$
Implementation of patient centricity within biopharmaceutical industry development programmes requires a consistent and coordinated approach, ${ }^{20}$ with a common definition and associated principles to ensure medicines deliver the optimal experience and outcome for patients and their loved ones. In this respect, it is important to ensure that the guiding principles of patient centricity are defined by patients for patients so that these can be used within industry as a point of reference and promote stronger unified commitment to patient engagement. Here, we describe the process leading to a consensus definition of patient centricity and its important principles impacting the biopharmaceutical industry as relates to the biopharmaceutical industry and the drug development process.

\section{METHODS}

\section{Establishing values of importance to patients}

As a first step in the process to define patient centricity and its important principles, AstraZeneca conducted an extensive multifaceted research project across key stakeholders to identify issues of importance to patients, healthcare providers and payers. Key questions to be answered through the various work streams were formulated by AstraZeneca and adapted where appropriate to elicit feedback from participants or to identify appropriate existing feedback from published (eg, Ernst and Young proprietary reports) and unpublished (AstraZeneca and Ernst and Young in-house data) sources (box 1). Participants were also given the opportunity to provide unsolicited feedback. Individual patients were identified, and together with patients recruited to an online forum (CreateHealth, see below), were invited to participate in accordance with appropriate ethical guidelines. Questionnaires were used to elicit feedback during telephone interviews with individual patients $(n=14)$ including those affiliated to patient organisations, and with patients $(n=200)$ through the online forum (CreateHealth proprietary crowd-sourcing healthcare platform) in the form of a virtual advisory board. Patients were recruited to the open (not gated) online forum using a

Box 1 Key questions used to establish values of importance to patients

How are wider consumer needs, behaviours and expectations changing?

- What implications do these changes have for organisations?

- What do patients, carers and patient organisations value when being engaged?

- What can we learn from providers and payers about being more patient centric? 
variety of digital recruitment tactics (eg, emails, banners, etc) across a range of social platforms (eg, Twitter, Facebook) and healthcare platforms (Patient Organisations, Health Unlocked). Further information was gathered from published and unpublished (AstraZeneca and Ernst and Young) sources. Patient feedback was further augmented with specific information offered by healthcare providers and payers $(n=5-10)$ relating to patient needs during telephone interviews.

\section{Defining patient centricity and the values of importance to patients}

Having gained a deeper understanding of patient needs and armed with key topics of interest from this early research, AstraZeneca conducted two identical workshops to create a definition of patient centricity and the healthcare values important to patients and caregivers (referred to as patients). A total of 22 patients or caregivers were identified either directly or through patient organisations and were invited to participate in these workshops in London, UK ( $n=10$ from across Europe), and then in Gaithersburg, USA $(n=12$ from across North America). Participants had been affected by a variety of illnesses (including anxiety, asthma, Asperger's syndrome, cancer (various), Crohn's disease, depression, diabetes, organ transplantation, psychiatric disease, rare disease). Some of these conditions were deliberately outside the scope of the AstraZeneca drug development strategy, as the company wished to understand the views and behaviours of a cross-section of patients, unrestricted by therapy area. All patients provided consent to participate. They were split into two groups (at each site) and asked to define patient centricity and the healthcare values most important to them. Preliminary definitions defined at both advisory boards were assimilated by the US attendees into a final version that was subsequently circulated to attendees of both advisory boards for their critique/confirmation.

\section{Validating the values of importance to patients}

The principles of importance were then tested through the PatientsLikeMe online patient network, the majority of whose contributors are located in North America. Using an online survey, patients were asked to score each of the patient-centric principles (previously defined) on a scale of 1 to 10 , to select their top three principles and provide any additional qualitative feedback. Given the greater North American focus of the PatientsLikeMe exercise, the principles were also tested among patients recruited from five European countries (France, Germany, Italy, Spain, UK) through the Carenity online patient network. The same questionnaire (but translated for local use) and analysis approach was used. No participant selection procedure was employed for the online surveys.

\section{RESULTS}

Establishing values of importance to patients

Patients reported that they can access a vast amount of conflicting health-related information, and that a critical part of being patient centric is to support access to easily understandable information through the channels they wish to use. Patients felt they need support to make the right decisions regarding their health, and offered that once patient needs are understood they are more open to positive influence. In this respect, the biopharmaceutical industry must rebuild trust and develop open long-term engaging relationships with patients. Access and affordability of medication and support is seen to be a key driver of patient engagement, and provision of a joined-up, system-wide approach to healthcare would provide an opportunity to improve patient experience. Patients also felt that they had to be a 'pain in the neck' to get the treatment they wanted and that the healthcare system should support patients to manage themselves. Payers and healthcare providers underscored that improvements in patients' health should be viewed holistically, and that improved relationships with other groups such as nurses, pharmacists and patient advocates are needed to meet patient needs more effectively. In addition, open and transparent access to product information, educational materials and clinical trial data are needed. Lack of patient compliance with treatment plans and fragmented payment systems were also seen as problematic.

Several key implications for the biopharmaceutical industry emerged. Efforts should be made to use communication channels of choice for the patient, and closer relationships should be developed to understand patient experiences, needs and preferences. The biopharmaceutical industry should also provide greater support for patients to achieve the best outcomes, taking a system-wide approach to patient access, including information and care. The industry and other healthcare providers should also help empower patients through the development and provision of self-management tools and services. The payer/provider perspective should also employ a more holistic approach to engaging patients, healthcare providers and payers, ensure transparency especially in relation to data, and work to improve systems and relationships in turn designed to improve health outcomes.

\section{Defining patient centricity and the values of importance to patients}

Definitions centred around four key areas defined as education and information, cocreation, access and transparency (table 1).

Four variations of a definition were created by the workshop participants which were then combined by the North America attendees to create the most appropriate representative statement (figure 1). This 
Table 1 Values/issues identified as being most important to patients (in their own words)

1. Education and information

Patient advocates and patient stories

Helping patients manage their own health and make their own decisions

Resources for families, carers and communities

Resources for healthcare professionals

Collect patient views and provide information about side effects

\section{Cocreation}

Work with patients and other stakeholders throughout the research, development and launch of medicines

Research patient's wider needs

Work with patients and other stakeholders to advocate policies in the interests of patients

Group of stakeholders to codesign solutions

\section{Access}

Support service to help patients navigate complex health systems and issues

Patient assistance programme

Flexible pricing policy

4. Transparency

Transparency with clinical trial data

When developing medicines, report on the measurable patient benefit and patient safety

Respond to patients' feedback — show how you have listened

Open and accountable reporting of progress

Values-based approach to business development

EU, European Union; NA, North America.
'Advocates related to each disease'. (NA)

'Educate patients through others' experience'. (NA)

'Support for patient education and patient "ambassadors" who can assist others'. (EU)

'Publish specialist (information) in conditions'. (NA)

'Customised info/data'. (EU)

'Support patients on treatments by providing information, insight, peer groups'. (EU)

'Supporting people to live healthy lives and sharing resources to benefit patients'. (EU)

'Young creative approach to awareness ... Online portal for meds, lifestyle, diet, health tips'. (EU)

'Knowing consequences to make an informed decision'. (EU)

'Support and guidance on how I can live with my condition (it's not just the medical side that matters - it has a financial impact, impact on the family, etc)'. (EU)

'Plain English'. (NA)

'Plain scientific language'. (EU)

'Patient-centred medicines leaflet developed by a Pharma company in Germany looked at what patients wanted to know, not just what the pharma medics/regulatory people had to tell them' (EU)

'Educate-families and communities'. (NA)

'(Don't) ignore family/caregiver concerns'. (EU)

'Facilitating family education'. (NA)

'Create information that doctors can distribute'. (NA)

'Champion the change in medical and organisational culture'. (EU)

'Patient centricity is not being taught to medical students and therefore there's no incentive to

learn how to be patient centric'. (EU)

'Inadequate education of clinical staff and support personnel'. (NA)

'Include patient voice-side effects'. (NA)

'Unbiased reporting of effects of treatments'. (EU)

'What is a tolerable side effect?' (NA)

'Open engagement with stakeholders across drug development pipeline'. (NA)

'Clinical trial end points patient input'. (NA)

'Shared goals and outcomes'. (EU)

'Including the patient in research from the beginning (this will improve compliance too)'. (EU)

'Recognition of quality of life beyond the medical model'. (EU)

'Researchers talk to patients to discover unmet need from them'. (NA)

'Understand patient journey (ideally) begins and ends at their home and not with your product or organisations'. (EU)

'Spending time with patients and their families to understand their experience'. (EU)

'Join and lobby for patient rights regarding meds (not just industry)'. (NA)

'Encourage and support patient lobby movement'. (EU)

'Pharmaceutical companies could develop a cross-healthcare advisory group to help "cocreate" solutions instead of just sponsorship'. (NA)

'Create Public Service Agreements (PSAs) to educate'. (NA)

'Have patient advisors 24/7'. (NA)

'Access to right therapy and information to support decision-making'. (EU)

'Compassion programme so no one is left out'. (NA)

'Make products and services affordable and have fair and honest pricing'. (NA)

'Share research findings to advance overall research'. (NA)

'Trial results reported up to trial participants'. (NA)

'Unbiased reporting of all trials'. (EU)

'Create the safest and most efficacious drugs'. (USA)

'Value and respond to feedback'. (NA)

'Good communication. Follow-up with backup'. (NA)

'Better medium between industry and patients'. (NA)

'Listening and learning from patients takes new ways of working/training'. (EU)

'Longevity of relationship not just project-focused'. (EU)

'An annual report of AZ's patient-centred activities'. (EU)

'Accountability from the AZ Board'. (EU)

'Measuring (patients') influence and impact on $A Z$ '. (EU)

'An action plan with measures'. (NA)

'Accountable for actions'. (NA)

'Socially responsible'. (NA)

'Balanced and ethical value proposition between profits and patients'. (NA) 


\begin{tabular}{|c|c|}
\hline Europe & North America \\
\hline $\begin{array}{l}\text { Every person is included } \\
\text { individual patient }^{2}\end{array}$ & $\begin{array}{l}\text { Educate patients and caregivers }{ }^{3} \text { in a culturally-sensitive } \\
\text { way }^{4} \text { to access the health and care system. Implement a } \\
\text { standard of excellence for all stakeholders }^{1} \text { in the health } \\
\text { and care system which provides respectful, accountable } \\
\text { and transparent } \\
\text { needs care that meets individual's rights and } \\
\text { neer }\end{array}$ \\
\hline $\begin{array}{l}\text { Where empathetic, responsible, sustained } \\
\text { innovative partnership }{ }^{4} \text { leads to where } \\
\text { everyone matters } \\
\text { ev }^{1} \text { for the ongoing benefit of } \\
\text { the patient }^{2}\end{array}$ & $\begin{array}{l}\underline{\text { Open }}^{4} \text { and continued engagement } \\
\text { caregivers, healthcare providers, pavers and the } \\
\text { biopharmaceutical industry }^{1} \text { to share knowledge } \\
\text { respect and compassion }^{4} \text { to offer the best possible } \\
\underline{\text { outcome for that person and their family }}^{2}\end{array}$ \\
\hline
\end{tabular}

In all cases, the definitions were shaped by the values that participants had originally identified as being most important to patients; ${ }^{2}$ Inclusiveness; ${ }^{2}$ Sharing goals that are patient and family-centred; ${ }^{3}$ Empowering patients to take control of their own health; ${ }^{4}$ Working in a way that shows respect, compassion and openness; ${ }^{5}$ Working in partnership.

\section{Putting the patient first in an open and sustained engagement of the patient to respectfully and compassionately achieve the best experience and outcome for that person and their family}

Figure 1 Patient centricity-definition evolution.

was circulated to all participants and received unanimous support. Patient centricity was thus defined as: 'Putting the patient first in an open and sustained engagement of the patient to respectfully and compassionately achieve the best experience and outcome for that person and their family'.

This definition encompassed five clear themes of importance: (1) inclusiveness; (2) sharing goals that are patient and family-centred; (3) empowering patients to take control of their own health; (4) working in a way that shows respect, compassion and openness; and (5) working in partnership (figure 1). Key words in the definition were underscored by more detailed descriptions. Thus, 'open and sustained engagement' reflects the need to act transparently in all interactions with patients and to maintain consistent relationships rather than those that are driven solely by project needs. 'Respectfully' reflects the need to foster a mutual understanding between both parties and to understand sometimes diverse viewpoints. 'Compassionate' underscores the need to empathise with a person, their condition and the impact it has on their lives, but at the same time recognising the valuable contribution that patients contribute in terms of healthcare decisions. 'Best experience and outcome' engenders the concept of supporting the whole person (rather than just the condition) through therapeutic and non-therapeutic means and understanding that all outcomes are unique for an individual. This requires an appreciation of the impact that each individual outcome has on patient's families, friends and the wider community and the need to respect patient's definitions of a desired outcome.

Ten principles were identified of importance to patients and caregivers for biopharmaceutical companies that centred around the previously defined key areas of education and information, cocreation, access and transparency (box 2).

\section{Validating the values of importance to patients and caregivers}

A total of 479 patients with cancer $(n=91)$, respiratory, inflammation or autoimmune disease $(n=246)$ or cardiovascular or metabolic conditions $(n=142)$ participated in the initial validation exercise through the PatientsLikeMe network; respondents were mainly from the USA ( $n=320$ vs 159 non-US). Patients confirmed that all ten principles were important for a biopharmaceutical company to embody, although patient empowerment received the lowest scores. When participants listed their top three principles, the access principles were cited most frequently (54\% of respondents), followed by education and information principles focused on unbiased information (42\%) and informed decisions principles (38\%). Of the principles not listed, a small proportion of patients (4\%) raised the need to ensure open communication on adverse events and side effects. 
Box 2 Principles of importance to patients and caregivers*

- The company helps make sure that the people who need medicines have access to them.

- The company communicates transparent and unbiased information on your disease, treatment options, and available resources with care and compassion.

- The company provides easy-to-understand and convenient information in plain language because they understand that 'words matter'.

- The company helps you gain affordable access to their medications.

- The company equips you to make informed choices about your healthcare and your treatment options.

- The company listens and responds to your feedback with respect and humility.

- The company partners with you to innovate and measure impact and outcomes that are important to you.

- The company provides access to support programmes and resources to help you improve quality of life.

- Everything the company does begins with an understanding of your needs and experiences.

- The company helps empower you to help other patients and their families.

- *Principles are not ranked by importance.

The principles were also tested in 703 patients with cancer $(n=99)$, respiratory, inflammation or autoimmune disease $(n=192)$ or cardiovascular or metabolic conditions $(n=412)$ recruited from five European countries (France $n=256$; Germany $n=99$; Italy $\mathrm{n}=158$; Spain $\mathrm{n}=71$; UK $\mathrm{n}=119$ ) through the Carenity patient network. As with the PatientsLikeMe exercise, European patients confirmed that all 10 principles were important for a biopharmaceutical company to embody, although again patient empowerment received the lowest scores. When participants selected their top three principles, provision of education and information were cited most frequently (39$40 \%$ of respondents) followed by education and access (39\%). Of the principles not listed, most participant suggestions related to 'putting people first' (25\%), followed by 'clear information on side effects' (16\%) and then 'improve transparency/honesty' (14\%). Of note, the need for clear information on side effects was a facet highlighted by the North American and the European groups.

\section{DISCUSSION}

Patient centricity is becoming a core element of medicine development and value-based healthcare management. Health outcomes are dependent on patient engagement and there is a clear need for the biopharmaceutical industry to partner with patients in the discovery, research, development, distribution and access to medicines to bring about better outcomes. Numerous patient engagement initiatives have started in the last few years, involving regulatory bodies, patient advocate groups, healthcare providers and payers, and the biopharmaceutical industry. Further, much dialogue and discussion has arisen around patient centricity but despite this process of 'cocreation' gaining momentum, there is still much to do. A consistent definition of patient centricity as applied to drug development has not been published, and although definitions relating to other key stakeholders (eg, healthcare providers ${ }^{24}$ ) have been developed, these are not transferable to the drug development process. We need a consistent and coordinated approach within the biopharmaceutical industry.

Against this background, a consistent definition of patient centricity and its associated principles for the biopharmaceutical industry were cocreated by patients and industry representatives from AstraZeneca through an extensive consultation exercise. These principles are consistent with those identified by other groups. ${ }^{26}$ Our call is for the biopharmaceutical companies to adopt this definition and the important principles which can then be used as a reference point for patient engagement throughout the product life cycle. By incorporating the definition and principles into the drug discovery and development process and beyond, we aspire to achieve measurable and positive improvements in patient outcomes. Indeed, AstraZeneca has already begun the process as described with incorporation of patient insight, cocreation of solutions for patients' needs and implementing methods for measuring the effectiveness of this approach. In this respect, AstraZeneca has embarked on a programme to ensure patient centricity is at the heart of medicine development and associated support/educational services. Overall, the patient-centric programme is designed to address patients' needs and to empower patients to connect patients with their providers, their community and other patients. It is intended to be sustainable and scalable, dynamic and to evolve as patient engagement continues. Ultimately, it is designed to have a measurable positive impact on patients' lives. Incorporating patient insight is an iterative process that can begin during early medicine development and continue throughout continuum of drug development. Patient insights can be used to inform disease area strategies, product development, clinical protocols, regulatory interactions and patient services.

We employed a comprehensive process to identify the principles of importance for patient engagement and to cocreate the definition of patient centricity. Although relatively small patient groups were engaged to refine these points, validation of the principles of importance was obtained from a larger cohort. The definition of patient centricity did not undergo 
further validation but AstraZeneca implemented patient centricity programmes on the basis of this definition. The research was also conducted in Europe and the USA and therefore may not be transferable to other geographical regions or where there are different healthcare models.

A call to action is for the biopharmaceutical industry and patients to consider the definition of patient centricity and principles of commitments to patients outlined in this paper and develop an action plan for demonstrating how they are living up to the principles engendered therein. Adopting this type of patient charter that clearly defines patient centricity and the associated principles could help further embed patient-centred practice. By partnering with patients, for patients, this new paradigm is more likely to deliver medicines that enable patients and their loved ones to benefit from the best possible experiences and outcomes.

In summary, we have cocreated principles of importance for patient engagement and a definition of patient centricity which provide an opportunity for biopharmaceutical companies to adopt and use these facets as a reference point for patient engagement throughout the product life cycle.

\begin{abstract}
Acknowledgements Medical writing support was provided by David Peters of Sequoia Medical Communications and funded by AstraZeneca. The authors would like to thank the patients and caregivers who participated in the live workshops held in the UK and USA. Background work and surveys to help define the issues of importance to patients were conducted by Ernst and Young and CreateHealth, sponsored by AstraZeneca. Surveys to validate the principles of importance to patients were conducted and analysed by PatientsLikeMe and Carenity, sponsored by AstraZeneca. The authors would like to thank the members of the PatientsLikeMe, Carenity and other patient communities who participated in these surveys.
\end{abstract}

Contributors All authors contributed to design, collection of data or interpretation of the results reported herein, and all authors contributed to the drafting, review and approval of the manuscript prior to submission.

Funding AstraZeneca.

Competing interests GY, HC and VG are employees of AstraZeneca. RRMJ is an ex-employee of AstraZeneca.

Provenance and peer review Not commissioned; externally peer reviewed.

Open Access This is an Open Access article distributed in accordance with the Creative Commons Attribution Non Commercial (CC BY-NC 4.0) license, which permits others to distribute, remix, adapt, build upon this work noncommercially, and license their derivative works on different terms, provided the original work is properly cited and the use is non-commercial. See: http://creativecommons.org/licenses/by$\mathrm{nc} / 4.0 /$

\section{REFERENCES}

1 Rago L, Santoso B. Drug regulation: history, present and future. In: Drug benefits and risks: international textbook of clinical pharmacology. 2nd edn. van Boxtel CJ, Santoso B, Edwards IR, eds. IOS Press and Uppsala Monitoring Centre, 2008.

2 International Conference on Harmonization of Technical Requirements for Registration of Pharmaceuticals for
Human Use 2015. http://www.ich.org/home (accessed Sep 2016).

3 Health Insurance Portability and Accountability. Standards for privacy of individually identifiable health information DHHS [45 CFR Parts 160, $162 \& 164$ (HIPAA)]. 2016 http://www.hhs.gov/hipaa/for-professionals/privacy/ (accessed Sep 2016).

4 European Medicines Agency. The patient's voice in the evaluation of medicines. How patients can contribute to the assessment of benefit and risk. $18^{\text {th }}$ October 2013. http://www. ema.europa.eu/docs/en_GB/document_library/Report/2013/10/ WC500153276.pdf (accessed Jan 2017).

5 Patient and Public Involvement In Research. HealthTalk.Org 2016. http://www.healthtalk.org/peoples-experiences/ improving-health-care/patient-and-public-involvement-research/ what-patient-and-public-involvement-and-why-it-important (accessed Sep 2016).

6 Global Genes. Uniting patients and caregivers, researchers, industry and government regulators at the table. A white paper expanding the panel discussion at the Global Genes Third Annual RARE patient's advocacy summit. March 2015. https://globalgenes.org/wp-content/uploads/2015/03/ Drug-Development-White-Paper-2015-FINAL.pdf (accessed Sep 2016).

7 Schaeffer S, McAllister E, Usdin S. Back to school: changing the subject. BioCentury $7^{\text {th }}$ September 2015. http://www. biocentury.com/Data/StaticContent/ContentFiles/090715_ Cover_BTS.pdf (accessed Jan 2017).

8 Hoos A, Anderson J, Boutin M, et al. Partnering with patients in the development and lifecycle of medicines: a call for action. Ther Innov Reg Sci 2015;49:929-39.

9 Brown MT, Bussell JK. Medication adherence: WHO cares. Mayo Clin Proc 2011;86:304-14.

10 Stevenson-Grund A. Listen to reason: patient reasons for medication noncompliance. Orbis Biosciences March $13^{\text {th }}$, 2015. http://www.orbisbio.com/blog/listen-to-reason-patientreasons-for-medication-noncompliance (accessed Jan 2017).

11 Robbins DA, Curro FA, Fox CH. Defining patient-centricity: opportunities, challenges, and implications for clinical care and research. Ther Innov Reg Sci 2013;47:349-55.

12 Ernst and Young. Progressions: health care everywhere. Global Life Sciences Report 2012 http://www.ey.com/GL/en/Industries/ Life-Sciences/Progressions-2012-Health-care-everywhereOverview (accessed Sep 2016).

13 Food and Drug Administration. Patient-Focused Drug Development: Disease Area Meetings Planned for Fiscal Years 2013-2017. 2015. http://www.fda.gov/ForIndustry/ UserFees/PrescriptionDrugUserFee/ucm347317.htm (accessed Sep 2016).

14 Food and Drug Administration. FDA Voice. FDA Announces First-ever Patient Engagement Advisory Committee. September 18, 2015. 2015. http://blogs.fda.gov/fdavoice/index.php/2015/ 09/fda-announces-first-ever-patient-engagement-advisorycommittee/ (accessed 5 Apr 2016).

15 Food and Drug Administration. Externally-Led Patient-Focused Drug Development Meetings. A Notice by the Food and Drug Administration. 28 December 2015. 2015c. https:/www. federalregister.gov/articles/2015/12/28/2015-32476/ externally-led-patient-focused-drug-development-meetings (accessed Sep 2016).

16 European Medicines Agency. Press Release, 9 December 2015. http://www.ema.europa.eu/docs/en_GB/document_library/ Press_release/2015/12/WC500198366.pdf (accessed Sep 2016). 
17 European Medicines Agency. News Release, 21 September 2015. http://www.ema.europa.eu/ema/index.jsp?curl=pages/ news_and_events/news/2015/09/news_detail_002394. jsp\&mid=WC0b01ac058004d5c1 (accessed Sep 2016).

18 European Union Medicines Agencies Network Strategy to 2020. Working together to improve health. 17 December 2015 EMA/MB/151414/2015. http://www.ema.europa.eu/docs/en GB/document_library/Other/2015/12/WC500199060.pdf (accessed Sep 2016).

19 Muhlbacher AC. Patient-centric HTA: different strokes for different folks. Expert Rev Pharmacoecon Outcomes Res 2015;15:591-97.

20 Perfetto EM, Burke L, Oehrlein EM, et al. Patient-focused drug development: a new direction for collaboration. Med Care 2015;53:9-17.

21 Vaida B. Patient-centred research outcomes: early evidence from the field. Health Aff 2016;35:4595-602.

22 Furlong P, Bridges JFP, Charnas L, et al. How a patient advocacy group developed the first proposed draft guidance document for industry for submission to the U.S. Food and Drug Administration. Orphanet J Rare Dis 2015;10:82
23 Boutin M, Dewulf L, Hoos A, et al. Culture and process change as a priority for patient engagement in medicines development. Ther Innov Reg Sci 2017;51:29-38.

24 Department of Health. Liberating the NHS; no decision about me, without me. $20^{\text {th }}$ July 2012 Available at: https:// consultations.dh.gov.uk/choice/choice-future-proposals/ supporting_documents/Choice $\% 20$ consultation $\% 20 \% 20 \mathrm{No} \%$ 20decison\%20about\%20me\%20without\%20me.pdf (accessed Jan 2017).

25 IMS Consulting Group. The idea of Patient Centricity is becoming a significant strategic focus across all healthcare stakeholders. But what does this term really mean? What does success look like? And are life science companies succeeding? http://www.imshealth.com:90/files/web/Global/ Services/Strategy\%20\&\%20Management\%20Consulting/ Consulting\%20Group\%20TL/IMS_Patient_Centricity.pdf (accessed Jan 2017).

26 National Health Council. The patient voice in value: The National Health Council Patient-centered value model rubric. March 2016. http://www.nationalhealthcouncil.org/sites/ default/files/Value-Rubric.pdf (accessed Jan 2017). 